\title{
ALGORITHM FOR INTEGRATED NON-DESTRUCTIVE DIAGNOSTICS OF TECHNICAL CONDITION OF STRUCTURES OF BUILDINGS AND CONSTRUCTIONS USING THE THERMOGRAM ANALYSIS
}

\author{
Denis $\mathrm{Karpov}^{1, *}$, and Anton Sinitsyn ${ }^{1}$ \\ ${ }^{1}$ Vologda State University, Lenina Street 15, Vologda, 160000, Russia
}

\begin{abstract}
An algorithm for integrated non-destructive diagnostics of technical condition of structures of buildings and constructions using the temperature field analysis is proposed. An attempt is made to systematize, characterize and build a logical sequence of stages for energy inspection of heat-consuming and heat-generating construction objects and their structural elements. The presented algorithm differs from the well-known technologies for energy monitoring of buildings and structures by the presence of two additional steps, which are an integrated part of any energy examination: determination of the relative surface humidity of building structures and the actual mass flow of coolant through a heating system using qualitative and quantitative assessment of thermal images. The implementation of energy (thermal imaging) and technical examinations according to the presented structural scheme requires appropriate equipment, as well as involvement of highly qualified specialists (civil engineers, thermal power engineers, thermal physicists, thermal imaging operators, etc.).
\end{abstract}

\section{Introduction}

The President and the Government of the Russian Federation set a strategic task for society and the state to determine ways to rationally use the country's most important national heritage - natural energy resources $[1$, 2].

Nowadays, the policy of energy and resource conservation is one of the priority economic areas for the entire national economy. Reducing the energy consumption of residential and public buildings, as well as various industrial facilities will create conditions for increasing the utilization efficiency of fuel, energy, material resources during production of heat, electricity, and a wide range of construction, industrial, and transport products. In order to take measures to optimize the energy consumption of a building or construction, it is essential to be able to make a qualitative and quantitative assessment of its current energy state. As for the thermal component, thermal imaging inspection has become widely used [3-6] for analysis of thermal state of objects of energy consumption and power generation, due to its efficiency and remote testing. Infrared thermography allows one to obtain the temperature image of the radiation surface of the object being examined (fig. 1).

Temperature is a universal way to show the state of an energy-consuming and energy-generating object. Temperature field characterizes the mechanical defects of building, the quality of hydraulic, electrical and other engineering equipment [3].

Thermal imaging has a great potential for assessing the energy state of residential buildings and industrial enterprises. It identifies defects at the initial stage of their appearance, thereby allowing one to determine and plan the scope and timing of repair, reconstruction,
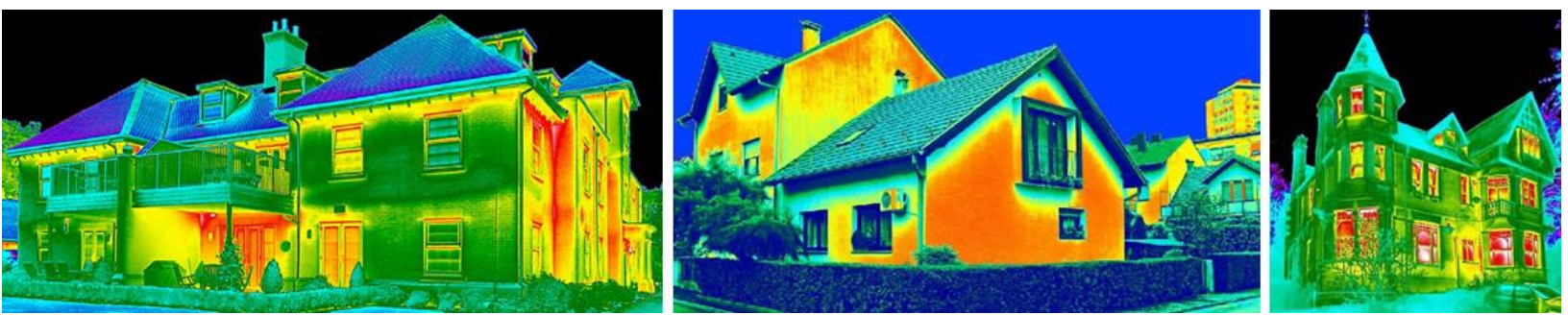

Fig. 1. Examples of thermograms of enclosing structures of buildings and constructions.

\footnotetext{
* Corresponding author: karpov_denis_85@mail.ru
} 


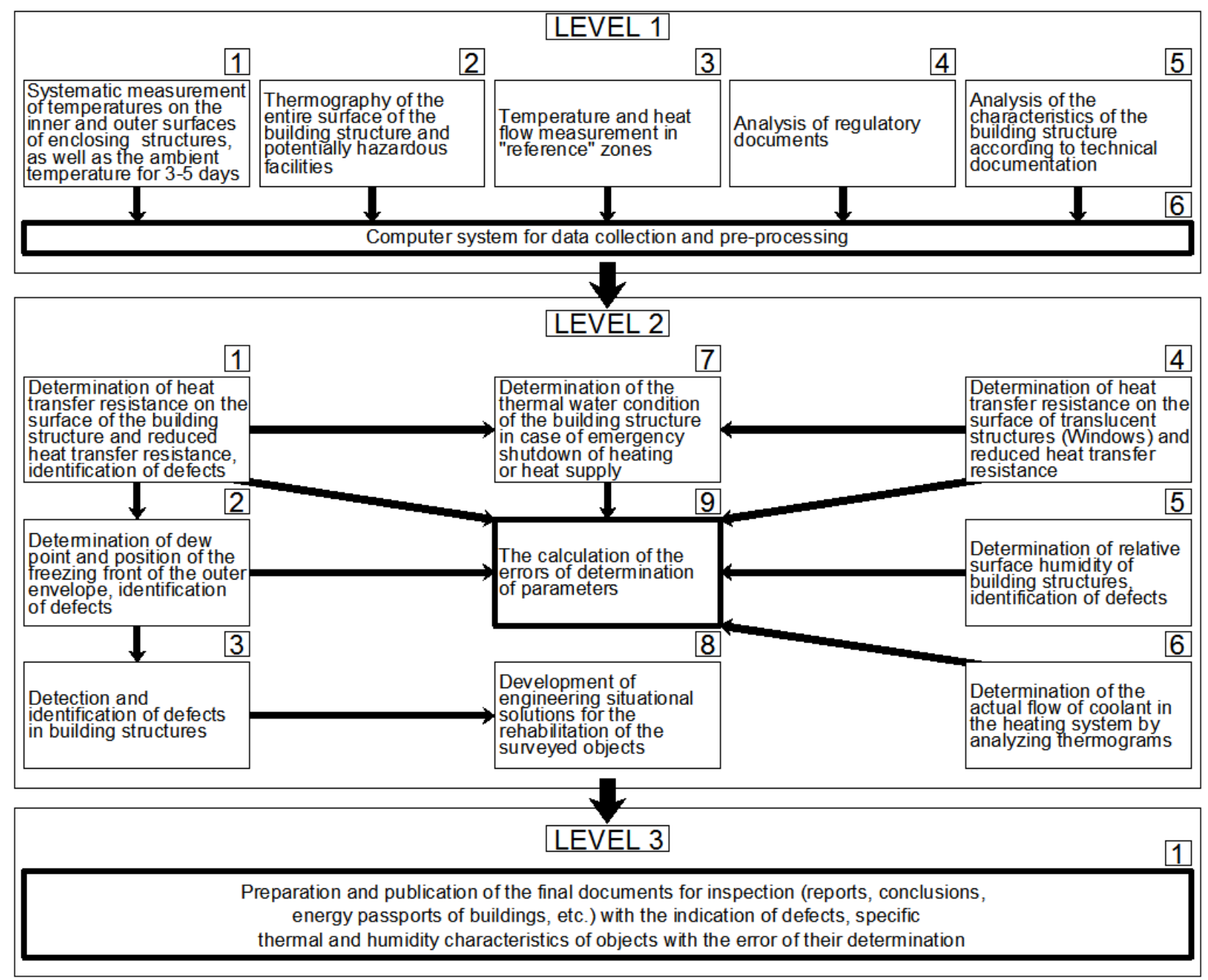

Fig. 2. The block diagram of the integrated non-destructive testing of technical condition of structures of buildings and constructions using the thermogram analysis.

modernization works. Thermal imaging is particularly valuable as this diagnostics is carried out in a nondestructive way without removal of controlled objects from operation [4].

Fig. 2 presents the structural diagram of the integrated non-destructive testing of technical condition of structures of buildings and constructions using the thermogram analysis.

Consider in more detail measures for integrated nondestructive monitoring of technical condition of structures of buildings and constructions, which are recommended to perform at each of the three levels presented (fig. 2).

Level 1 consists of 6 sequential (parallel) stages. The first five stages are finished by acquisition and preliminary computer processing of the obtained data:

1). Systematic measurements of temperatures of the internal and external surfaces of enclosing structures, as well as of ambient temperature (outdoor and/or indoor air) for 3-5 days.

2). Thermal imaging of the entire surface of building structure and potentially hazardous facilities.
3). Measurements of temperature and heat flows in "reference" zones.

When performing the first three stages, one should use the equipment presented in detail in [3, 4]: thermocouples, thermal imager, pyrometer, thermohygrometer, hot-wire anemometer, heat flux density meter, etc.

4). A detailed analysis of regulatory documents on thermal control, construction, energy, etc.

5). Analysis of the main characteristics (geometric, technical, physical and mechanical, etc.) of a building structure according to the technical documentation.

Examples of computer systems for acquisition and preliminary computer processing of the obtained data are presented in detail in [3, 4].

Level 2 consists of 9 sequential (parallel) stages:

1). Determination of heat transfer resistance over the surface of a building structure and reduced heat transfer resistance over the thickness of the enclosing structure. The technique for determining this value is well known and described in detail in [7, 8]. Downward deviation of the actual (calculated, operational) heat transfer resistance of the building structure from the required 


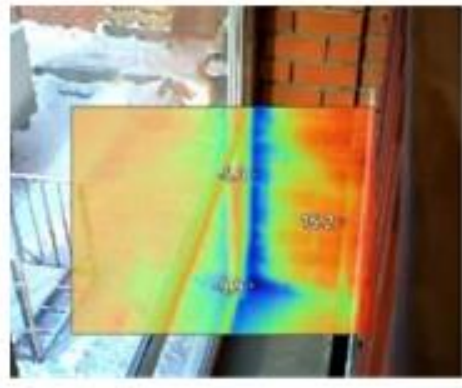

a

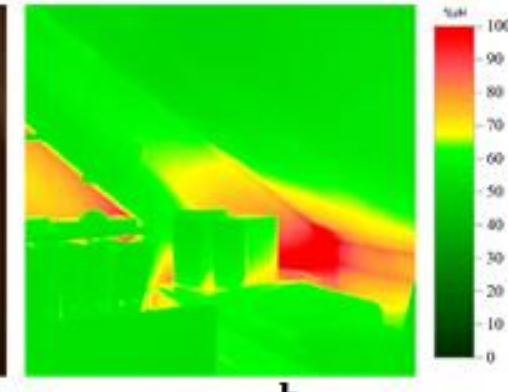

b

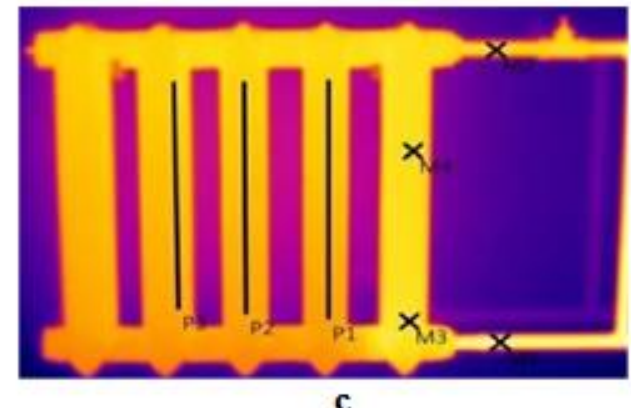

C

Fig. 3. Examples of thermograms with heat and humidity defects of various enclosing structures and engineering equipment.

(normative) value of this parameter indicates insufficient thermal protection of the enclosing structure, and/or the presence of a thermal defect (thermal temperature anomaly) and/or technical defect. In general, a thermal defect is understood as a local change in the distribution of thermal radiation of the controlled object and is interpreted as a deviation from the norm. A technical defect can be a hidden shell, cavity, crack, various foreign inclusions, etc., as well as various deviations of physical properties of the controlled object from the norm, the place of local overheating (cooling), etc. Defects are also classified as active and passive. Defects that emit or absorb thermal energy, and are diagnosed (identified) in a passive mode of thermal control, are called active.

2). Determination of dew point and the position of the freezing front of the outer enclosing structure. Modern thermal imaging equipment and related specialized software allow one to automatically calculate the dew point temperature. In order to do that, one should enter temperature and relative humidity of environment into the thermal imager $[3,4]$.

3). Detection and identification of thermotechnical defects of building structures [3-6].

4). Determination of heat transfer resistance along the surface of translucent structures (windows, transoms, lights, etc.) and reduced heat transfer resistance. Special attention should be paid to thermography of translucent structures of buildings and structures, since the main heat losses occur through them (fig. 3 a).

5). Determination of relative surface humidity of building structures and identification of defects. Defects in enclosing structures, improper operation of buildings and structures, poor-quality building materials and products with reduced heat and moisture protection properties can trigger an increase in indoor humidity, which will inevitably lead to the formation of biocorrosion. The methodology for determining the relative surface humidity of structures of buildings and constructions is also one of the capabilities of modern thermal imaging equipment and related specialized software. The technology allows one to visualize the relative surface humidity for each individual point of the thermogram (fig. 3 b).

6). Determination of the actual flow of coolant through the heating system using the analysis of thermograms (fig. 3 c). This value can be used to determine the actual coolant velocity in the connections to the heating device, hydraulic losses in pipelines, the degree of clogging of pipes and sections of the heating device, noise level of the hydraulic system, or when calculating the actual thermal power of the heating system section, etc. [9].

7). Determination of temperature and humidity conditions of the entire building structure in case of planned or emergency shutdown of heating or heat supply systems [10].

8). Development of engineering situational solutions for rehabilitation of the surveyed objects. The purpose of rehabilitation is to ensure both comfortable living conditions and compliance with modern regulatory requirements for heat and moisture protection, energy and resource conservation. In general, rehabilitation is understood as the complex of all types of reconstruction, modernization of buildings and structures carried out by special technology. A striking example of rehabilitation of construction sites is thermal insulation using various thermal insulation materials.

9). Calculation of error in determining the parameters in order to establish deviations of the measured values from their true (real) values.

Level 3 consists of 1 final stage:

1). Preparation and publication of final inspection documents (reports, conclusions, energy certificates of buildings, etc.) containing indication of defects, specific thermal and humidity characteristics of the objects with the error in their determination; measures to eliminate the identified technical, thermal, humidity defects; recommendations for increasing energy efficiency and rational use of energy resources.

The block diagram of the integrated non-destructive diagnostics of technical condition of structures of buildings and constructions based on the analysis of thermal images is proposed. The implementation of energy (thermal imaging) and technical inspections according to the presented scheme requires not only the availability of appropriate equipment, as well as involvement of highly qualified specialists (civil engineers, thermal power engineers, thermal physicists, thermographic operators, and other).

An attempt was made to systematize and logically build a sequence of stages of energy inspection of heatconsuming and heat-generating construction objects and their structural elements using the thermogram analysis. The described algorithm includes the main stages 
performed during such examinations. It differs from the known methods of energy monitoring of buildings and structures by the presence of two additional stages at level 2 (stages 5 and 6), which are an integral part of any energy survey.

\section{References}

[1] The energy strategy of Russia for the period until 2030: Resolution of the Government of the Russian Federation from 13.11.2009, 1715-r.

[2] The Federal Law "On Energy Saving and on Improving Energy Efficiency and on Amending Certain Legislative Acts of the Russian Federation", Reed Group LLC (2012).

[3] O.N. Budadin, V.P. Vavilov, E.V. Abramova, Thermal control: textbook, SPECTR Publishing House (2013).

[4] V.P. Vavilov, Thermal imaging and thermal control for engineers, The 1st edition, SPECTR Publishing House (2017).

[5] S. George, S. Goravar, D. Mishra, M.T. Shyamsunder, P. Sharma, G.K. Padmashree, P.S. Kumar, P. Bremond, K. Mukherjee, Stress monitoring and analysis using lock-in thermography, Insight, 52, 9 (2010).

[6] G.K. Vijayraghavan, M.C. Majumder, K.P. Ramachandran, NDTE using flash thermography: numerical modelling and analysis of delaminations in GRP pipes, Insight, 52, 9 (2010).

[7] K.F. Fokin, Construction heat engineering of enclosing parts of buildings, The 5th ed., revised, AVOK-PRESS (2006).

[8] Y.V. Shefer, O.A. Antonevich, B.S. Ordobaev, S.V. Romanenko, Conception of low-rise earthquake-resistant energy-efficient buildings, IOP Conf. Series: Materials Science and Engineering, 81 (2015).

[9] D.F. Karpov, Possible options for quantitative assessment of thermal images, Prirodoobystroistvo, 1 (2019).

[10] Yu.M. Varfolomeev, O.Ya. Kokorin, Heating and heating networks: textbook, INFRA-M (2008). 